\title{
Three Junction Holographic Micro-Scale PV System
}

\author{
Yuechen $\mathrm{Wu}^{*}{ }^{* a}$, Shelby Vorndran ${ }^{\mathrm{b}}$, Silvana Ayala Palaez ${ }^{\mathrm{a}}$, Raymond K. Kostuk ${ }^{\mathrm{a}, \mathrm{b}}$
}

The University of Arizona: ${ }^{a}$ Department of Electrical and Computer Engineering, 1230 E Speedway Blvd. Tucson AZ 85721; ${ }^{b}$ College of Optical Sciences, 1630 E. University Blvd. Tucson, AZ 85721

\begin{abstract}
In this work a spectrum splitting micro-scale concentrating PV system is evaluated to increase the conversion efficiency of flat panel PV systems. In this approach, the dispersed spectrum splitting concentration systems is scaled down to a small size and structured in an array. The spectrum splitting configuration allows the use of separate single bandgap PV cells that increase spectral overlap with the incident solar spectrum. This results in an overall increase in the spectral conversion efficiency of the resulting system. In addition other benefits of the micro-scale PV system are retained such reduced PV cell material requirements, more versatile interconnect configurations, and lower heat rejection requirements that can lead to a lower cost system. The system proposed in this work consists of two cascaded off-axis holograms in combination with a micro lens array, and three types of PV cells. An aspherical lens design is made to minimize the dispersion so that higher concentration ratios can be achieved for a three-junction system. An analysis methodology is also developed to determine the optical efficiency of the resulting system, the characteristics of the dispersed spectrum, and the overall system conversion efficiency for a combination of three types of PV cells.
\end{abstract}

Key worlds: Solar energy, Spectrum splitting, Multi-junction PV, Micro-Scaled Photovoltaic, holography, concentrating photovoltaics

\section{INTRODUCTION}

Solar energy can be a competitive renewable energy source when it provides high optical-to-electrical power conversion efficiency and energy yield at low cost. The efficiency of a single junction PV device is limited, because photons with energy different from the bandgap energy can lead to loss. Multiple Junction (MJ) PV system can significantly improve conversion efficiency by managing the solar spectrum with multiple bandgaps ${ }^{1}$. In addition, concentrating PV (CPV) designs can improve the conversion efficiency by increasing the open circuit voltage ${ }^{2}$. A practical high efficiency PV design must utilize both direct and diffuse solar illumination conditions since even locations with high direct normal incidence (DNI) have significant levels of diffuse illumination ${ }^{3,4}$.

The typical Multi-Junction Concentrating PV (MJCPV) system usually utilizes optical focusing elements to concentrate solar irradiance into a small area of tandem PV cells. Although MJCPV can obtain high efficiency under standard test conditions, a few issues exist in such a design. First, the performance of tandem PV cells is limited by the bandgap with the lowest current and is optimized for standard solar spectrum. As a result, the systems using tandem MJ PV cells can suffer from significant loss in efficiency when operating under actual operating conditions ${ }^{5}$. Next, the acceptance angle of an optical CPV system is restricted by the conservation low of etendue. This limits the system conversion ability for diffuse irradiance and reduce its overall conversion efficiency in northern regions. Finally, depending on the focusing power, the typical CPV design usually requires large vertical separation between the optical collector and PV cell location. This increases the installation cost and prevents use for many applications such as rooftop system.

The Sandia National Laboratory first developed the micro-scale concentrating (MSC) PV systems ${ }^{6-8}$. Their system scales a typical large CPV into micro size, and integrates them in to arrays. With this design, the system can be formed as a flat panel, and have "smart" interconnection between PV cells. In addition, integrated circuit fabrication methods can dramatically reduce manufacturing costs. During the same time Yamada and Okamoto proposed a CPV design to collect both direct and diffuse illumination ${ }^{9}$. The design consists of a typical MJ cell at the focus of a Fresnel lens in conjunction with silicon cell surrounding the MJ cell. This resulted in a significant increase in output with both direct (1.19X) and diffuse (37.3X) illumination conditions.

In this research, a three-junction holographic spectrum splitting MCPV system was studied and incorporates many of the features discussed above. The system design was targeted to have high conversion efficiency with direct irradiance and maximized efficiency with diffuse illumination for different geographical locations and spectrum conditions. A methodology for evaluating system performance is also developed and presented in this work.

*ywu2@email.arizona.edu

Next Generation Technologies for Solar Energy Conversion VII, edited by Oleg V. Sulima,

Gavin Conibeer, Proc. of SPIE Vol. 9937, 99370M · (C) 2016 SPIE

CCC code: $0277-786 \mathrm{X} / 16 / \$ 18 \cdot$ doi: $10.1117 / 12.2237004$

Proc. of SPIE Vol. 9937 99370M-1 


\section{HOLOGRAPHIC MICRO-SCALE SPECTRUM SPLITTING SYSTEM}

\subsection{System overview}

Volume holograms can be designed to diffract a specific spectral bandwidth with high diffraction efficiency (DE). In addition, they can be mass produced at low cost making them useful for practical spectrum splitting PV systems. In this paper, we proposed a three junction holographic spectrum splitting micro-scale concentrating PV (HSSMCPV) system to obtain high conversion efficiency under both direct and diffuse solar irradiance. This system consists of two transmission volume holograms placed above an aspherical lens array to direct different components of solar spectrum into the most efficient PV cells (Figure 1). The receiver plane consists of silicon ( $\mathrm{Si})$ cells, indium gallium phosphide ( $\mathrm{InGaP}$ ) cells, and gallium arsenide ( $\mathrm{GaAs}$ ) cells arranged horizontally. To achieve high $\mathrm{DE}$ under direct illumination, the two holograms were designed with different diffraction angles for InGaP and GaAs cells. The lens array was optimized to minimize aberration at the diffraction angles. The smaller GaAs and $\mathrm{InGaP}$ cells are surrounded by Si cells for diffuse light capture.

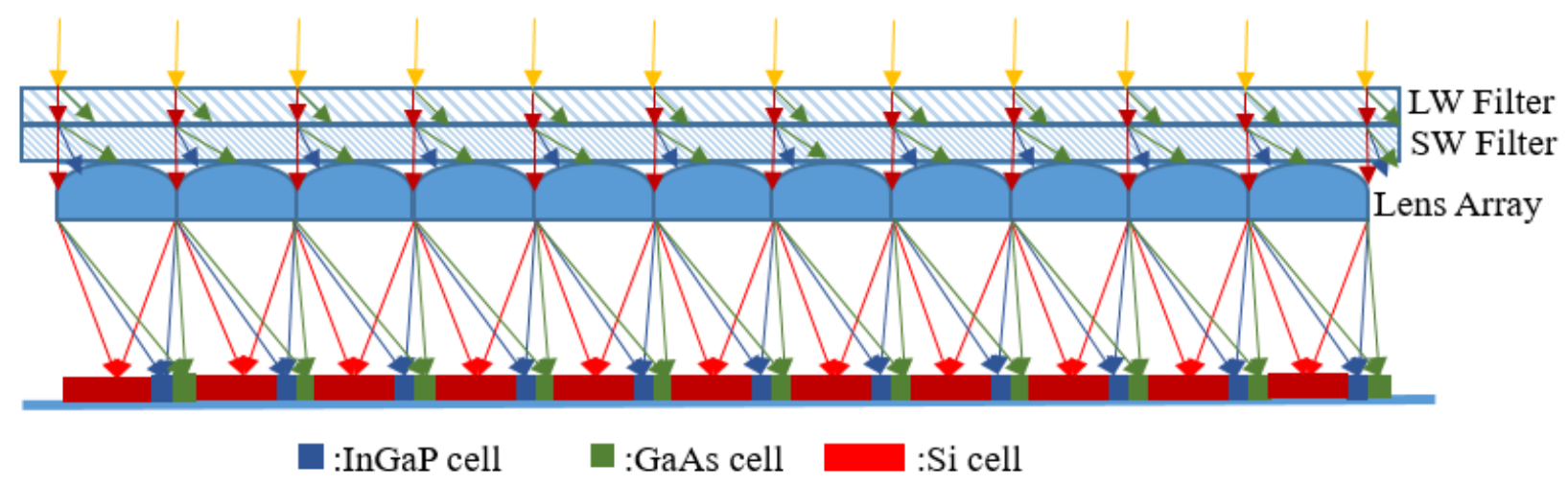

Figure 1. The 2D structure of the proposed three-junction HSSMCPV system.

\subsection{Design procedure}

The design of an HSSMCPV system depends on several parameters: 1) the types of PV cells to be used; 2) the system geometry; 3) transmission volume hologram characteristics including the diffraction angles, the peak diffracted wavelength, and peak diffraction efficiency; and 4) the lens design for the lens array.

\subsubsection{Cell selection}

In spectrum splitting PV systems, the high conversion efficiency is obtained by using multiple PV cells that have the highest spectral conversion efficiency (SCE) over a broad spectral bandwidth. For an M-junction spectrum splitting PV system, the maximum combined efficiency under spectral solar irradiance $(\mathrm{E}(\lambda))$ can be expressed as:

$$
\eta_{\max }=\frac{1}{P} \sum_{k=1}^{M} \int_{-\infty}^{+\infty} E(\lambda) S C E_{k}(\lambda) d \lambda,
$$

where, $\mathrm{P}$ is the total incident power; and $S C E_{k}(\lambda)$ is the SCE of the kth PV cell.

For this design, a $24.7 \%$ efficient silicon cell, a $22.6 \%$ InGaP cell, and a $26.9 \%$ GaAs cell were used with performance parameters selected from the literature ${ }^{10-13}$. Figure 2 shows the calculated SCE of InGaP, GaAs, and Si cells overlaid with the AM1.5G solar spectrum. The SCE and conversion efficiency of each cell were calculated based on their external quantum efficiency (EQE), open circuit voltage, and fill factor under standard test conditions obtained from published data. These PV bandgaps were chosen because of their high conversion efficiency, and SCE advantage. As shown in Figure 2., the InGaP cell has the highest SCE for wavelengths between $400 \mathrm{~nm}$ to $675 \mathrm{~nm}$, the GaAs cell between $675 \mathrm{~nm}$ and $875 \mathrm{~nm}$, and the Si cell between $875 \mathrm{~nm}$ and $1200 \mathrm{~nm}$. The bandgaps for the $\mathrm{InGaP}$ and GaAs cells determine the transition and peak efficient wavelengths for the holographic filters. 


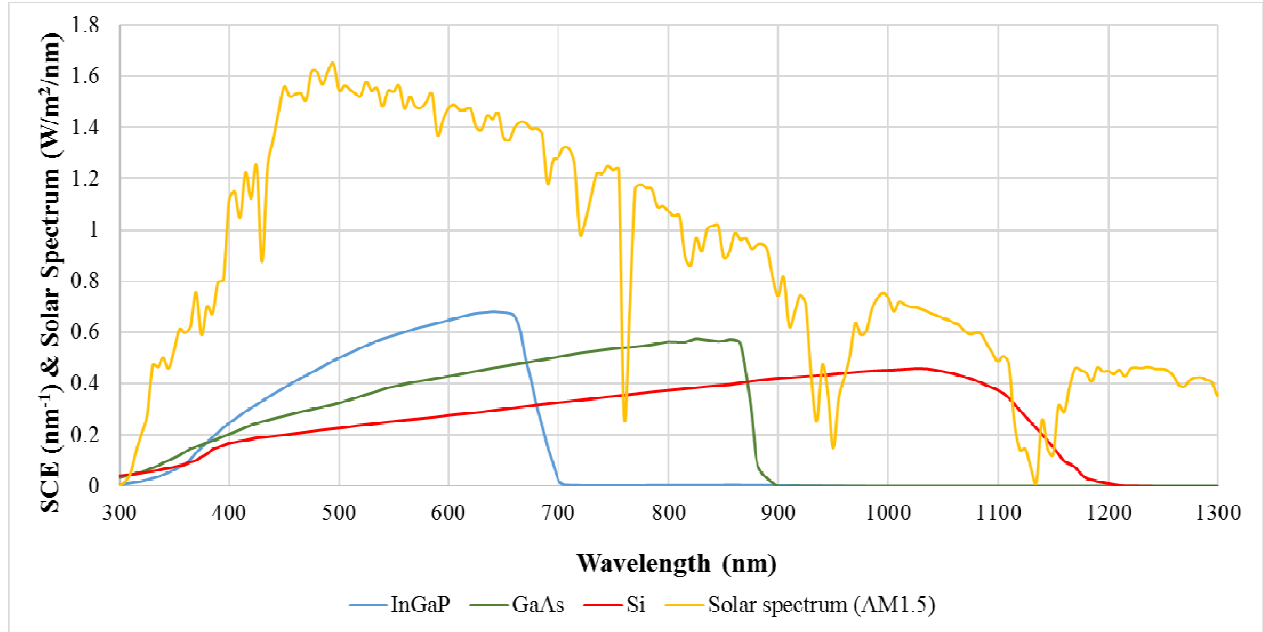

Figure 2. Spectral conversion efficiency (SCE) of InGaP, GaAs and Si cells selected for the spectrum-splitting module, and the AM1.5 solar spectrum.

\subsubsection{System geometry}

In the three-junction HMSSPVS, the hologram filters are used to split spectral components of the incident direct solar illumination into different diffraction angles, and the lens array concentrates the different spectral components into the corresponding PV cells as shown in Figure 3 (a).

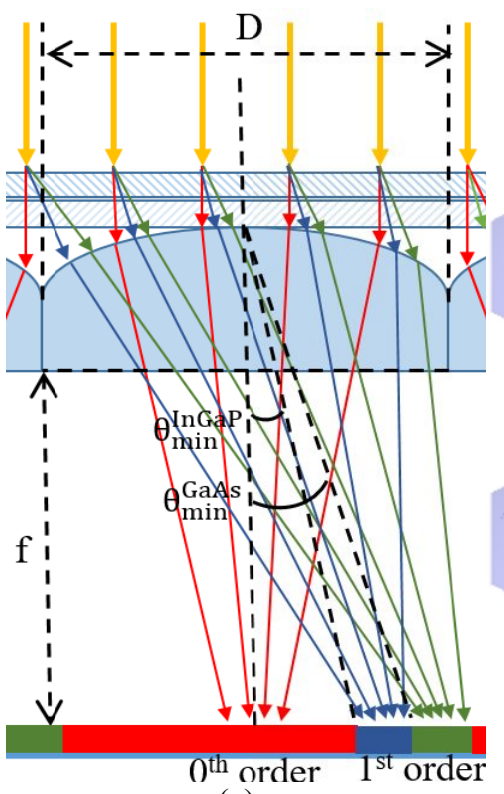

(a)
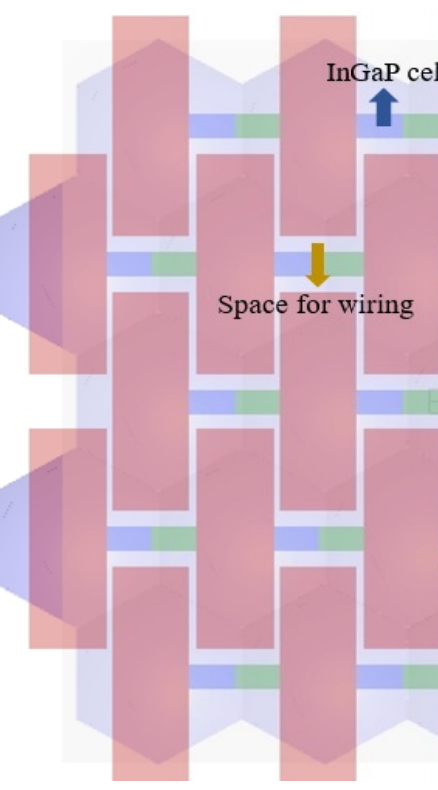

(b)

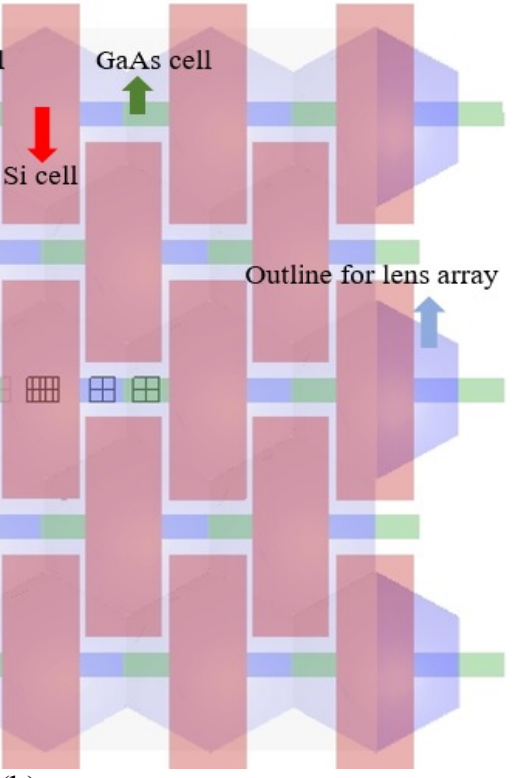

Figure 3. (a) Spectrum splitting functionality of a unit element of the array, and (b) the top view of the PV cell arrangement of the HMSSCPV system. The light blue hexagons are the outline of the micro lenses; the blue, green and rad pads are the InGaP, GaAs, and $\mathrm{Si}$ solar cells; and the space in between the cells are left for wiring purpose.

The design includes several considerations. First, in each unit element, to optimize the overall conversion efficiency, the lowest bandgap PV cell ( $\mathrm{Si}$ ) is placed at the $0^{\text {th }}$ order, and the higher bandgap PV cells (InGaP and GaAs) are placed at off-axis positions on the receiver plane. The two transmission holograms incorporate with the lens diffract and focus the corresponding first order beams to the GaAs and InGaP cells. Light not directed to the InGaP and GaAs cells, including the desired spectral bandwidth for silicon cell and the desired diffraction spectral bandwidth that remains at the $0^{\text {th }}$ order, can be collected by the silicon cells. 
Next, as will be discussed in Section 2.2.3, a minimum diffraction angle is required to obtain high volume hologram diffraction efficiency in a single diffraction order. In this design, the transition wavelengths for the short wavelength filter (SWF) and the long wavelength filter (LWF) are 400nm, 675nm (InGaP), and $675 \mathrm{~nm}, 875 \mathrm{~nm}$ (GaAs). The minimum diffraction angles for the shortest diffraction wavelengths in each filters are $7.79^{\circ}$ at $400 \mathrm{~nm}$ and $10.1^{\circ}$ at $675 \mathrm{~nm}$. Since the holograms are embedded in glass, the two angles increased to $11.7^{\circ}$ and $15.25^{\circ}$ in air. To ensure that the large diffraction angles in each unit structure do not interfere with the adjoining units, the lens elements in the array must have high focusing power and low aberrations.

Another design consideration is the dispersion of the diffracted solar illumination. It has been shown that sharp transitions between two wavelength bands can be obtained with high concentration ratio (focusing) optics ${ }^{14}$. In this design, two cascaded holographic filters were used to improve the transition properties between InGaP and GaAs cells. The top hologram (LWF) was designed to diffract the longer wavelengths into larger diffraction angles for GaAs cells, and the bottom hologram(SWF) was designed to diffract the shorter wavelength band into smaller diffraction angles for InGaP cells.

Finally, the size and the arrangement for each PV cell was adjusted to optimize the optical efficiency. For each unit element of the array (Figure 3), the size of InGaP and GaAs cells were reduced to small rectangular shapes that only collect the corresponding focused wavelengths, while the size of Si cells was maximized to collect the $0^{\text {th }}$ order and the diffuse irradiance. Some narrow space was also set between each unit elements to allow for wiring connections (Figure 3 (b)).

A summary of design parameters based on the above principles is given in Table 1.

Table 1. The geometric and optical parameters of the three-junction HMSSCPV system for the primary design.

\begin{tabular}{|c|c|c|c|c|}
\hline \multicolumn{3}{|c|}{ Hologram parameters } & \multicolumn{2}{|c|}{ Geometric parameters } \\
\hline & LWF & SWF & Lens diameter & $15 \mathrm{~mm}$ \\
\hline Peak wavelength & $525 \mathrm{~nm}$ & $775 \mathrm{~nm}$ & Focal length & $15 \mathrm{~mm}$ \\
\hline Diffraction band & $400 \mathrm{nn}-650 \mathrm{~nm}$ & $650 \mathrm{~nm}-890 \mathrm{~nm}$ & Si cell size & maximized \\
\hline $\begin{array}{l}\text { Diffraction angle for } \\
\text { transition wavelength }\end{array}$ & $15^{\circ}(400 \mathrm{~nm})$ & $25^{\circ}(650 \mathrm{~nm})$ & InGaP cell size & $\begin{array}{l}\text { Minimized for best optical } \\
\text { efficiency }\end{array}$ \\
\hline Hologram thickness & $22 \mu \mathrm{m}$ & $22 \mu \mathrm{m}$ & GaAs cell size & $\begin{array}{l}\text { Minimized for best optical } \\
\text { efficiency }\end{array}$ \\
\hline
\end{tabular}

\subsubsection{Holographic element design}

Several considerations were included in the design of the transmission volume holograms: 1) the grating period along the surface is chosen based on the diffraction angle for the desired transition wavelength. 2) the grating period in the depth direction is chosen based on the desired peak efficient wavelength and its diffraction efficiency (DE); and 3) the hologram material properties including index modulation, film thickness and differential swelling conditions are chosen based on the desired spectral bandwidth of the diffracted beam.

The holographic filters are designed for normal incident light. The desired lateral (surface) grating period of each grating can be calculated for the first order diffraction angle, $\theta_{t}$, at the transition wavelength, $\lambda_{t}$, using the grating equation:

$$
\sin \left(\theta_{t}\right)=\frac{\lambda_{t}}{n \Lambda_{x}}
$$

where $\mathrm{n}$ is the average refractive index in the medium, and $\Lambda_{x}$ is the lateral grating period of the grating.

Once the diffraction angle is obtained for the transition wavelength, the lateral grating period is determined by equation (2). To obtain high DE at the desired peak efficient wavelength, $\lambda_{\text {peak }}$, the vertical period $\left(\Lambda_{z}\right)$ is required to satisfy the Bragg condition ${ }^{15}$ :

$$
\vec{K}=\overrightarrow{k_{1}}-\overrightarrow{k_{2}}=\frac{2 \pi}{\Lambda_{x}} \hat{x}+\frac{2 \pi}{\Lambda_{z}} \hat{z}=\frac{2 \pi n}{\lambda_{\text {peak }}}\left\{\sin \left(\theta_{\text {peak }}\right) \hat{x}+\left[1-\cos \left(\theta_{\text {peak }}\right)\right] \hat{z}\right\}
$$

where, $\vec{K}$ is the grating vector; $\overrightarrow{k_{1}}$ and $\overrightarrow{k_{2}}$ are the wave vectors of the incident and diffracted beams, and $\theta_{\text {peak }}$ is the diffraction angle of the peak efficient wavelength. 
For normal incident beam, the grating period component in the depth direction, $\Lambda_{z}$, can then be determined according to:

$$
\begin{aligned}
& \Lambda_{x}=\frac{\lambda_{\text {peak }}}{n \sin \left(\theta_{\text {peak }}\right)}, \\
& \Lambda_{z}=\frac{\lambda_{\text {peak }}}{n\left[1-\cos \left(\theta_{\text {peak }}\right)\right]} .
\end{aligned}
$$

Also, in order to achieve high diffraction efficiency in a single diffraction order the physical parameters of the volume grating must satisfy the condition:

$$
\mathrm{Q}=\frac{2 \pi \lambda d}{n \Lambda^{2}} \geq 10,
$$

where $\lambda$ is the operating wavelength, $d$ is the thickness of the hologram; $n$ is the average refractive index of the hologram; and $\Lambda$ is the volume grating period. This parameter is often referred to as the Kogelink Q parameter ${ }^{14}$. The minimum diffraction angle, $\theta_{\min }$, that satisfies the volume diffraction condition can be calculated by:

$$
\theta_{\min }=2 \arcsin \left(\sqrt{\frac{5 \lambda}{4 \pi n d}}\right)
$$

The physical parameters for dichromated gelatin (DCG) were considered for holographic filter material. This material provides high refractive index modulation and has long-term stability properties ${ }^{16}$. The film thickness can be controlled during the film deposition process and sets the basic spectral bandwidth. Further modification to the shape and width of the diffracted spectral bandwidth can be achieved by varying the film processing method. The refractive index modulation as a function of depth can be controlled by varying the film composition, recording wavelength, and film processing method.

\subsubsection{Lens design}

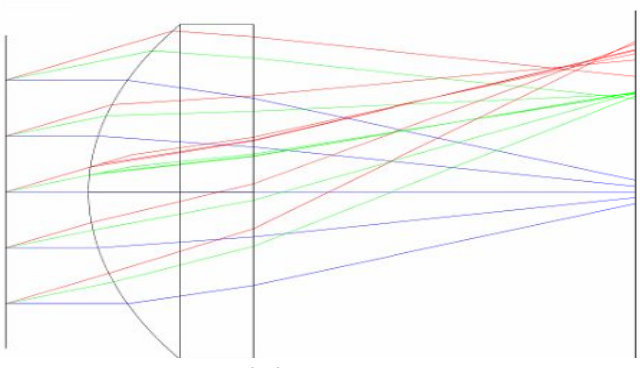

(a)

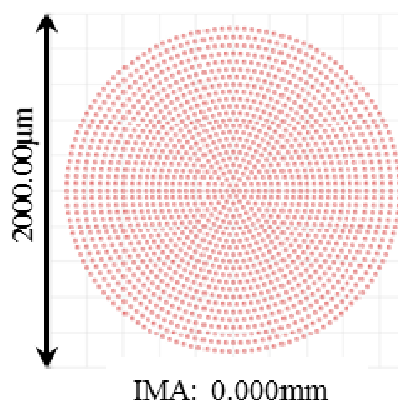

(b)

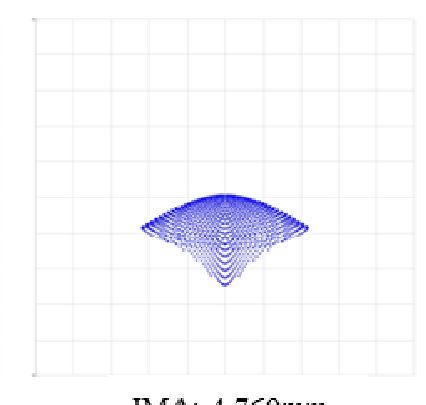

IMA: $4.769 \mathrm{~mm}$

(c)

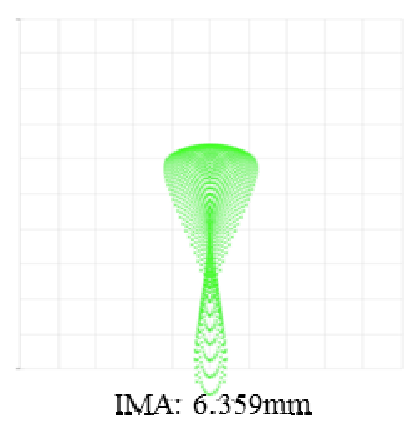

(d)

Figure 4. (a) The 2D plot of the designed aspherical lens. The spot diagram b) is for normally incident light at 1000nm; c) is for light incident at $18^{\circ}$ at $450 \mathrm{~nm}$; and d) is for light incident at $25^{\circ}$ at $700 \mathrm{~nm}$. The same scale is used for the three spot diagrams.

The lens elements in the array are required to have high focusing power, low aberration, and high optical efficiency for a range of incident angles. An aspherical lens was chosen for the design because the optical aberrations, in particular 
spherical aberration, can be controlled with a single surface element. PMMA was selected for the material for the lens array due to its low cost and good optical properties. The lens was designed by optimizing the $2^{\text {nd }}, 4^{\text {th }}, 6^{\text {th }}$, and $8^{\text {th }}$ order aspherical coefficients of the focusing surface using Zemax ${ }^{17}$. Figure 4(a) shows the optical layout and spot diagrams for an optimized F/1 with a focal length of $15 \mathrm{~mm}$. The final receiver plane was placed at $13.8 \mathrm{~mm}$ away from the back surface of the lens to minimize the spot size at InGaP (Figure 4 (c)) and GaAs (Figure 4 (d)) cells. The focused spot area for silicon cell (Figure 4 (b)) is allowed to be large, since it is also used for diffuse light collection.

\subsection{System efficiency}

The SCE can be used as a metric for comparing PV cells for spectrum splitting systems ${ }^{19}$. In a CPV system, the high intensity that exists at the PV cell surface increases the $\mathrm{V}_{\mathrm{oc}}$ and the SCE over that of a one sun system. This increase on the intensity can be quantified as the quantum concentration ratio which can be defined as:

$$
X_{Q}=\frac{I_{L X}}{I_{L 1}}=\frac{X_{G} \int_{-\infty}^{+\infty} E(\lambda) \eta_{o p t}(\lambda) S R(\lambda) d \lambda}{\int_{-\infty}^{+\infty} E(\lambda) S R(\lambda) d \lambda}
$$

where $X_{Q}$ is the quantum concentration ratio, $I_{L X}$ is the current generated under concentration, $I_{L 1}$ is the current generated under one sun conditions, $X_{G}$ is the geometrical concentration ratio defined as the area of system entrance aperture divided by the PV cell area, $E(\lambda)$ is the solar spectral irradiance, $\eta_{\text {opt }}(\lambda)$ is the spectral optical efficiency (i.e. the spectral efficiency of the incident illumination transferred to a particular PV cell in the system), and $S R(\lambda)$ is the spectral response of the PV cell. In a single junction CPV system, since the spectral power distributions on the system entrance and the surface of PV cell are identical $\left(\eta_{\text {opt }}(\lambda)=1\right)$ and the quantum concentration ratio is equal to the geometric concentration ratio. However, in a SSCPV system, they are different.

For a PV cell in spectrum splitting CPV systems with a quantum concentration ratio of $X_{Q}$, the SCE can be expressed as:

$$
\operatorname{SCE}_{X}(\lambda) \cong S R(\lambda) * F F * V_{o c}^{X}=S R(\lambda) * F F *\left(V_{o c}^{S}+\mathrm{m} \frac{k T}{q} \ln \left(X_{Q}\right)\right)=\operatorname{SCE}_{S}(\lambda)+\mathrm{SR}(\lambda) * F F * \mathrm{~m} \frac{k T}{q} \ln \left(X_{Q}\right),
$$

where, the $\operatorname{SCE}_{X}(\lambda)$ and $\operatorname{SCE}_{S}(\lambda)$ are the spectrum conversion efficiencies under concentration ratio and standard test conditions, $V_{o c}^{X}$ and $V_{o c}^{S}$ are the open circuit voltages under concentration and standard test conditions, FF is the fill factor, $\mathrm{m}$ is the diode ideality factor, $\mathrm{k}$ is the Boltzmann's constant, $\mathrm{T}$ is the operation temperature and $\mathrm{q}$ is the electron charge.

The resulting overall conversion efficiency of an M-junction spectrum splitting CPV system can be calculate as:

$$
\eta_{\text {sys }}=\sum_{k=1}^{M} \frac{1}{P} \int_{-\infty}^{+\infty} E(\lambda) \eta_{k}(\lambda) S C E_{X}^{k}(\lambda) d \lambda,
$$

where $\mathrm{P}$ is the total incident optical power, $\eta_{k}(\lambda)$ is the overall optical spectral efficiency for the kth bandgap, and $S C E_{X}^{k}(\lambda)$ is the SCE of the kth bandgap cell in the system.

To evaluate the proposed three-junction HMSSCPV system, the optical spectral efficiency for each bandgap cell and the concentration ratios were determined using ratracing and diffraction efficiency analysis. These values were then combined with the computed values for $\operatorname{SCE}_{X}(\lambda)$ to determine the overall conversion efficiency of the system.

\section{DESIGN RESULTS}

\subsection{Holographic filters}

The DCG material considered for this design has an average refractive index of 1.57 , and index modulation up to 0.08 , a thickness $\mathrm{d}=$ $22 \mu \mathrm{m}$, and a differential swelling coefficient of 0.2 . These values can be achieved with typical DCG fabrication and processing methods ${ }^{16}$. The final gratings were designed and simulated by a rigorous coupled wave analysis (RCWA) software RSoft DiffractMOD $^{\mathrm{MT}} 18$. The refractive index modulation was varied for each grating to optimize the performance.

The grating periods for the LWF and SWF were pre-calculated using the method described on Section 2.2.2. To optimize the optical efficiency and the bandwidth, the grating performance has been simulated by varying the index modulation from 0.01 to 0.1 . Figure 5 shows the spectral diffraction efficiency of long wavelength (a) and short wavelength (b) filters for different index, and the optimized diffraction efficiencies as a function of wavelength for the two gratings (c). 


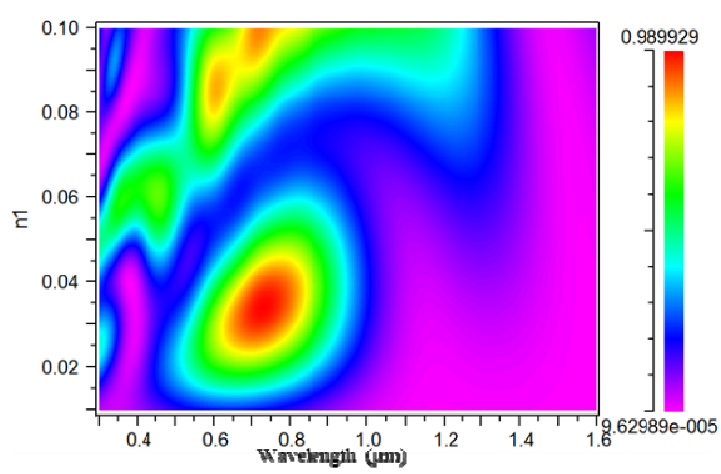

(a)

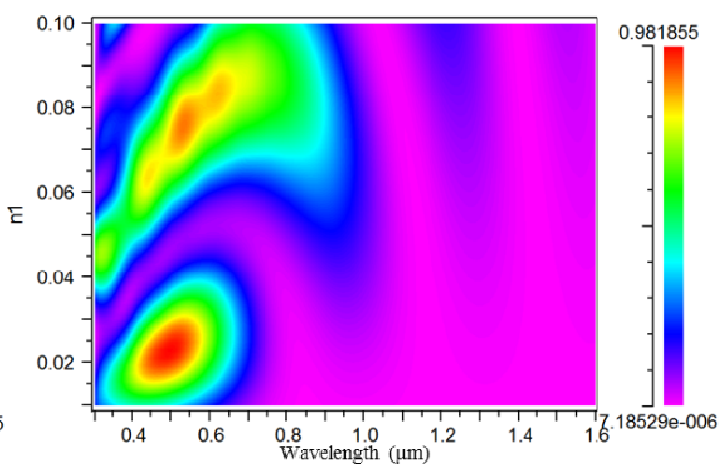

(b)

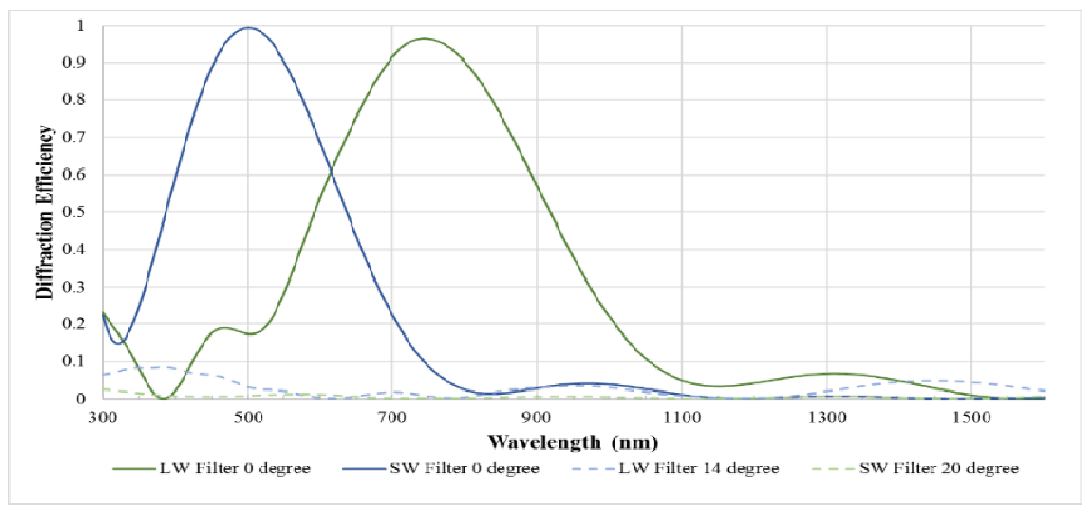

(c)

Figure 5. Diffraction efficiency (DE) as a function of refractive index modulation and wavelength for (a) the long wavelength filter and (b) the short wavelength filter. (c) The first order DE for the optimized filters under the normal incident (solid lines) and larger angle of incident (dashed lines). The low DE for the larger incident angles indicates that there is minimal interaction between diffracted beams for the cascaded filters.

\subsection{System parameters}

The design process described in section 2 was used to determine the system geometrical properties and are summarized in Table 2 .

Table 2. System parameters

\begin{tabular}{cc}
\hline Parameter & Value \\
\hline Holographic film thickness & $22 \mu \mathrm{m}$ \\
Refractive index modulation & $0.038(\mathrm{LWF})$ \\
Lens size for unit cell & $0.024(\mathrm{SWF})$ \\
System height (focal length) & Hexagon with a $=7.5 \mathrm{~mm}$ \\
Lens array f/\# & $15 \mathrm{~mm}$ \\
InGaP cell size & $\sim \mathrm{f} / 1$ \\
GaAs cell size & $1 \mathrm{~mm} \mathrm{X3.5} \mathrm{mm}$ \\
Si cell size & $1 \mathrm{~mm} \mathrm{X3.5} \mathrm{mm}$ \\
Geometric concentration on InGaP cell & $20 \mathrm{~mm} \mathrm{X6mm}$ \\
Geometric concentration on GaAs cell & $41.74 \mathrm{X}$ \\
Geometric concentration on Si cell & $41.74 \mathrm{X}$ \\
Quantum concentration on InGaP cell & $1.22 \mathrm{X}$ \\
Quantum concentration on GaAs cell & $29.2 \mathrm{X}$ \\
Quantum concentration on Si cell & $13.7 \mathrm{X}$ \\
& $0.52 \mathrm{X}$ \\
\hline
\end{tabular}




\section{MODELING AND SIMULATION RESULTS}

A model of the three-junction HMSSCPV system was designed and built using non-sequential ray tracing in the Photon Engineering FRED ${ }^{\circledR}$ Optimum simulation program ${ }^{20}$. The diffractive efficiencies of both the LWF and SWF for all wavelengths and incident angles were simulated using the RCWA in RSoft DiffractMOD ${ }^{\mathrm{MT}}$ and inserted in the nonsequential ray tracing model for the corresponding filter surfaces. The rays intercepting corresponding PV cell surfaces were weighted by their diffraction efficiency values and used to determine the system optical efficiency.

\subsection{Raytracing analysis}

The optical system shown in Figure 1 was analyzed using non-sequential raytracing as part of the optical efficiency analysis. A unit cell of the system consists of the two cascaded holographic filters, the aspheric lens, and a receiver plane at $13.8 \mathrm{~mm}$ from the back surface of the aspheric lens. The simulation was performed on a sub assembly of 5 X 5 unit cells as shown in Figure 6. Reflective surfaces were also used around the sub assembly to enhance optical efficiency.

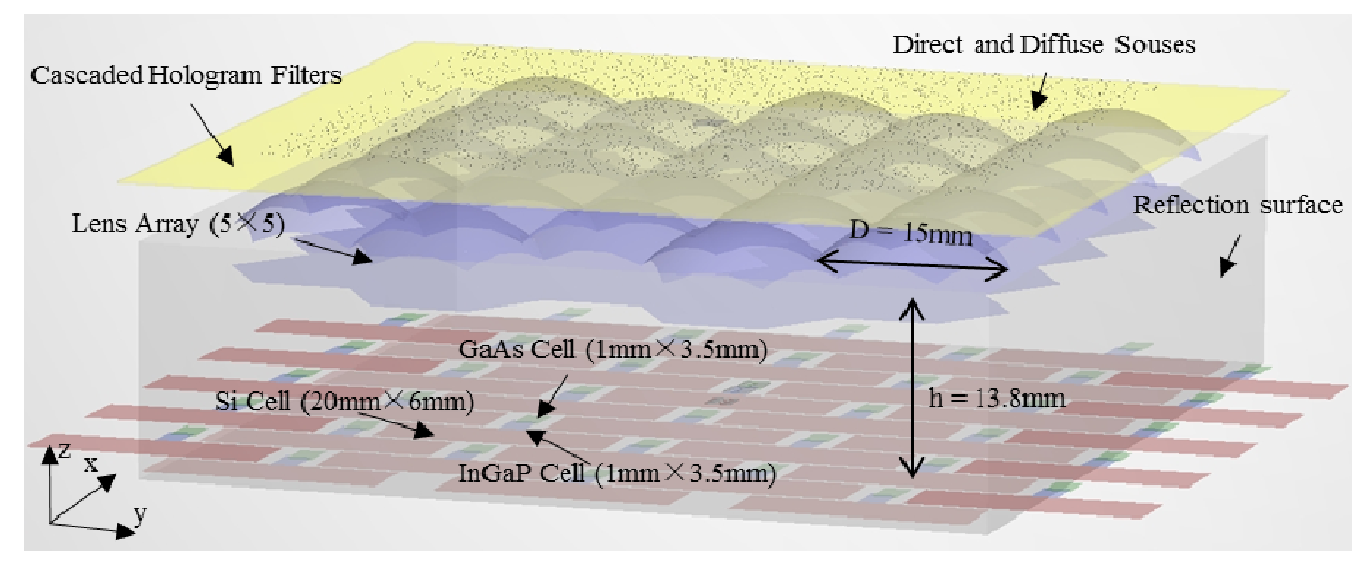

Figure 6. 3D FRED model for the three-junction HMSSCPV system.

The model allows the normal incident rays to be split into diffracted orders with an appropriate diffraction efficiency determined by rigorous coupled wave analysis. An example of the ray trace with three different spectral bandwidths is shown in Figure 7. On the receiver plane, three groups of absorbing surfaces were used to model the Si cells (red), InGaP cells (blue), and GaAs cells (green) are shown. During the simulation, the spectral optical power absorbed by each group of absorbing surfaces was separately analyzed to obtain the spectral optical efficiency of each PV cell type.

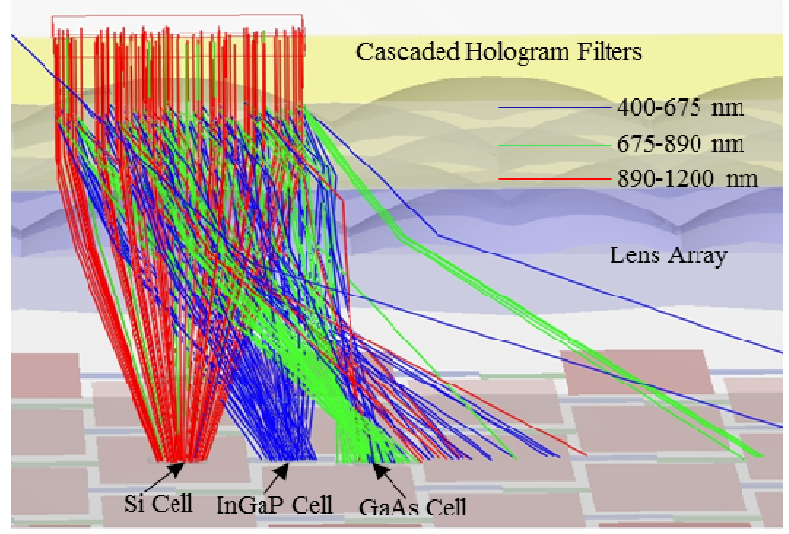

Figure 7. Raytrace simulation of the spectrum splitting for a single unit structure in the three-junction HMSSCPV system.

In the raytracing model, the direct and diffuse irradiance of AM 1.5 solar spectrum were simulated as a source plane in the system. The rays from the direct source were incident normally to the filters and have an AM1.5D spectrum and an irradiance of $900 \mathrm{~W} / \mathrm{m}^{2}$. The rays from the diffuse source were randomly incident over a range of angles from $-90^{\circ}$ to $+90^{\circ}$ and assigned with diffuse AM1.5 spectrum spectral illumination and $100 \mathrm{~W} / \mathrm{m}^{2}$. 


\subsection{Simulation results for optical, and power conversion efficiency}

The optical irradiance distribution at each PV cell surface was computed and is shown along with the direct AM1.5 spectrum in Figure 8. The loss in optical irradiance at the InGaP and GaAs cells is mainly due to power distribution in higher diffraction orders and diffraction away from the PV cell surfaces by the cascaded filters. However, a significant fraction of this power is captured by the Si cells.

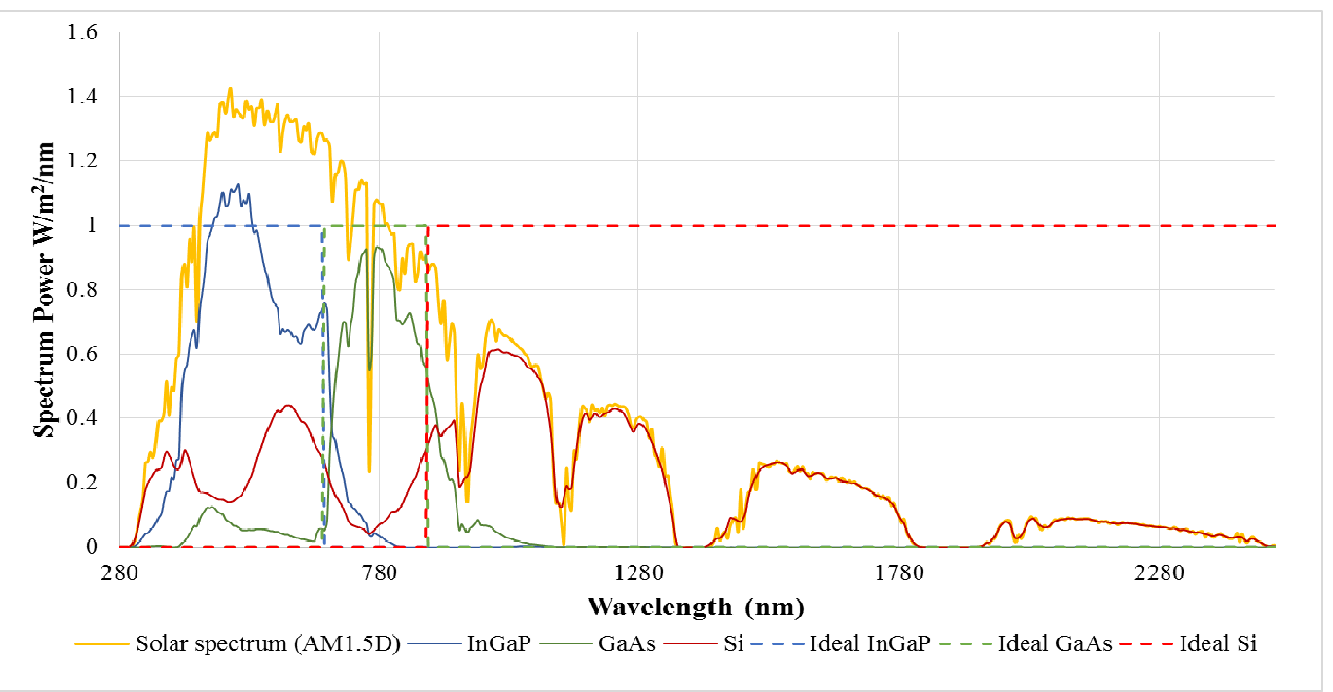

Figure 8. The simulated optical power distribution at each PV cell type compared with the AM1.5 direct spectrum. The dashed lines show ideal filter functions for each cell type.

The optical and power conversion efficiency of the HMSSCPV and the individual PV cells used in the system are given in Table 3. The optical efficiency at each cell surface with diffuse and direct AM1.5 illumination shows the significant contribution of the large area silicon cells. The power conversion efficiency columns show the large contribution of InGaP with direct illumination but a large drop in output from this cell with diffuse conditions.

Table 3. The simulation results of optical efficiency and power conversion efficiency for the HMSSCPV system under AM1.5 illumination.

\begin{tabular}{c|cc|ccc}
\hline & \multicolumn{2}{|c|}{ Optical Efficiency } & \multicolumn{3}{c}{ Power Conversion Efficiency } \\
\hline & AM1.5_Direct & AM1.5_Diffuse & AM1.5_Direct & AM1.5_Diffuse & AM1.5_Global \\
HMSSPVS & $91.14 \%$ & $71.29 \%$ & $\mathbf{3 3 . 7 3} \%$ & $\mathbf{1 7 . 5 1 \%}$ & $\mathbf{3 2 . 1 1 \%}$ \\
InGaP & $29.24 \%$ & $4.07 \%$ & $14.99 \%$ & $1.16 \%$ & $13.61 \%$ \\
GaAs & $19.98 \%$ & $3.92 \%$ & $9.74 \%$ & $1.16 \%$ & $8.88 \%$ \\
Si & $41.92 \%$ & $63.30 \%$ & $9.00 \%$ & $15.19 \%$ & $9.62 \%$ \\
\hline
\end{tabular}

Table 4. The efficiencies of single junction systems with the same aperture and involved PV cells under AM1.5D\&G (left), and the improvement of the HMSSCPV on conversion efficiency over each of the individual cell under AM1.5D\&G (right).

\begin{tabular}{c|cc|cc}
\hline & \multicolumn{2}{|c|}{ Single Junction Efficiency } & \multicolumn{2}{c}{ HMSSCPV Improvement/Single Junction } \\
\hline & AM1.5_Direct & AM1.5_Global & AM1.5_Direct & AM1.5_Global \\
InGaP & $22.00 \%$ & $22.64 \%$ & $53.32 \%$ & $41.82 \%$ \\
GaAs & $26.54 \%$ & $26.85 \%$ & $27.09 \%$ & $19.58 \%$ \\
Si & $24.76 \%$ & $24.69 \%$ & $36.23 \%$ & $30.04 \%$ \\
\hline
\end{tabular}

The left columns of Table 4 show the power conversion efficiency for the single junction cells used in HMSSCPV system with direct and global AM1.5 illumination conditions. The individual cells in this comparison have the same collection area as the HMSSCPV. The right columns of Table 4 show the power conversion efficiency improvement over the performance of the individual cells with direct and global AM1.5 illumination. The results show very significant improvement in conversion efficiency for all cases (19.58-53.32\%). 


\section{CONCLUSION}

A holographic micro-scale spectrum splitting concentrating system with three different PV cell bandgaps was designed and evaluated. The design using cascaded transmission volume gratings significantly improves the system conversion efficiency under direct solar irradiance. The Si cells as large part of the receiver surface also ensures the collection of diffuse solar irradiance. The periodic arrangement of the receiver plane provides a potential for integration and mass production to reduce the cost. The methodology for designing this HMSSCPV system includes the system geometric, holographic filter, and lens design was described in detail. The diffraction efficiencies of the holographic filters were modeled using RCWA software, and the optical performance of the HMSSCPV system was simulated with non-sequential raytracing software. Under the direct AM1.5 spectral illumination, the system reaches the optical efficiency of $91.14 \%$ and system conversion efficiency of $33.73 \%$, which indicates a $27.09 \%$ improvement over best bandgap (GaAs), a 36.23\% improvement over the major cell in the geometry (Si), and a 53.32\% over the major functioning cell (InGaP). Under diffuse AM1.5 illumination, the system has a conversion efficiency of $17.51 \%$ primarily resulting from the silicon cell output.

\section{ACKNOWLEDGEMENTS}

The authors wish to acknowledge support from the NSF/DOE ERC cooperative agreement No. EEC-1041895, NSF grant ECCS-1405619.

\section{REFERENCES}

[1] Polman, A. and Atwater, H. A., "Photonic design principles for ultrahigh-efficiency photovoltaics," Nat. Mater. 11(3), 174177 (2012).

[2] Mart'I, A. and Luque,A., [Next Generation Photovoltaics High efficiency through full spectrum utilization], Institute of Physics Publishing, Bristal \& Philadelphia, 108-119 (2004).

[3] "National Solar Radiation Data Base: 1991-2005 Update: Typical Meteorological Year 3," NREL. Retrieved 18, http://rredc.nrel.gov/solar/old_data/nsrdb/1991-2005/tmy3/, (16 July 2016).

[4] Vorndran, S., Russo, J., Zhang, D., Gordon, M. and Kostuk, R. K., "A radiometric method for optimizing CPV performance for site-specific irradiance conditions," Photovoltaic Specialists Conference (PVSC), 2012 38th IEEE, 002105-002110 (2012).

[5] Broderick, L. Z., Albert, B. R., Pearson, B. S., Kimerling, L. C. and Michel, J. , "Design for energy: Modeling of spectrum, temperature and device structure dependences of solar cell energy production," Solar Energy Mater.Solar Cells 136, 48-63 (2015).

[6] Furman, B., Menard, E., Gray, A., Meitl, M., Bonafede, S., Kneeburg, D., Ghosal, K., Bukovnik, R., Wagner, W., Gabriel, J., Seel, S. and Burroughs, S., "A high concentration photovoltaic module utilizing micro-transfer printing and surface mount technology," Photovoltaic Specialists Conference (PVSC), 2010 35th IEEE, 000475-000480 (2010).

[7] Jared, B. H., Saavedra, M. P., Anderson, B. J., Goeke, R. S., Sweatt, W. C., Nielson, G. N., Okandan, M., Elisberg, B., Snively, D., Duncan, J., Gu, T., Agrawal, G. and Haney, M. W., "Micro-concentrators for a microsystemsenabled photovoltaic system," Opt.Express 22(S2), A521-A527 (2014).

[8] Nielson, G. N., Okandan, M., Cruz-Campa, J. L., Resnick, P. J., Wanlass, M. W., Clews, P. J., Pluym, T. C., Sanchez, C. A. and Gupta, V. P., "Microfabrication of microsystem-enabled photovoltaic (MEPV) cells," Proc. SPIE 7927, Advanced Fabrication Technologies for Micro/Nano Optics and Photonics IV, 79270P (2011).

[9] Yamada, N. and Okamoto, K. , "Experimental measurements of a prototype high concentration Fresnel lens CPV module for the harvesting of diffuse solar radiation," Opt.Express 22(S1), A28-A34 (2014).

[10] Masuko, K., Shigematsu, M., Hashiguchi, T., Fujishima, D., Kai, M., Yoshimura, N., Yamaguchi, T., Ichihashi, Y., Mishima, T., Matsubara, N., Yamanishi, T., Takahama, T., Taguchi, M., Maruyama, E. and Okamoto, S., "Achievement of More Than 25\% Conversion Efficiency With Crystalline Silicon Heterojunction Solar Cell," IEEE Journal of Photovoltaics 4(6), 1433-1435 (2014).

[11] Geisz, J. F., Steiner, M. A., García, I., Kurtz, S. R. and Friedman, D. J. , "Enhanced external radiative efficiency for 20.8\% efficient single-junction GaInP solar cells," Appl.Phys.Lett. 103(4), 041118 (2013).

[12] Green, M. A., Emery, K., Hishikawa, Y., Warta, W. and Dunlop, E. D. , "Solar cell efficiency tables (version 42)," Prog Photovoltaics Res Appl 21(5), 827-837 (2013). 
[13] Kayes, B. M., Nie, H., Twist, R., Spruytte, S. G., Reinhardt, F., Kizilyalli, I. C. and Higashi, G. S., "27.6\% Conversion efficiency, a new record for single-junction solar cells under 1 sun illumination," Photovoltaic Specialists Conference (PVSC), 2011 37th IEEE, 000004-000008 (2011).

[14] Russo, J., Vorndran, S., Wu, Y., Kostuk, R.K., "Cross-correlation analysis of dispersive spectrum splitting techniques for photovoltaic systems," J. Photon. Energy. 5(1), 054599 (2015).

[15] Kogelnik, H.. "Coupled wave theory for thick hologram gratings," Bell Syst. Tech. J., 48(9), 2909-2947 (1969).

[16] Chang, B. J. and Leonard, C. D., "Dichromated gelatin for the fabrication of holographic optical elements," Appl. Opt., 18(14), 2407-2417 (1979).

[17] "OpticalStudio software", Zemax, LLC., <http://www.zemax.com/os/opticstudio> (16 July 2016).

[18] "DiffractMOD Product Overview," Synopsys, < https://optics.synopsys.com/rsoft/rsoft-passive-device-diffractMOD.html >. (16 July 2016)

[19] Russo, J. M., Zhang, D., Gordon, M., Vorndran, S., Wu, Y. and Kostuk, R. K., "Spectrum splitting metrics and effect of filter characteristics on photovoltaic system performance," Opt.Express 22(S2), A528-A541 (2014).

[20] “FRED Optimum software”, Photon Engineering, Inc., <http://www.photonengr.com/software/> (16 July 2016). 